\title{
Integrating hospital and community care: using a community virtual ward model to deliver combined specialist and generalist care to patients with severe chronic respiratory disease in their homes
}

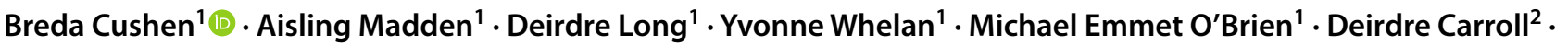 \\ Des O'Flynn ${ }^{2} \cdot$ Michelle Forde $^{2} \cdot$ Virginia Pye ${ }^{3} \cdot$ Loretto Grogan $^{3} \cdot$ Margaret Casey $^{3} \cdot$ Karolina Farrell $^{4}$. \\ Richard W. Costello ${ }^{1}$. Clare Lewis ${ }^{4}$
}

Received: 28 February 2021 / Accepted: 1 May 2021 / Published online: 6 May 2021

(c) Royal Academy of Medicine in Ireland 2021

\begin{abstract}
Background Chronic respiratory diseases are responsible for significant patient morbidity, mortality, and healthcare use. Community virtual ward (CVW) models of care have been successfully implemented to manage patients with complex medical conditions.

Aims To explore the feasibility and clinical outcomes of a CVW model of care in patients with chronic respiratory disease. Methods Patients known to specialist respiratory services with Chronic Obstructive Pulmonary Disease (COPD) and/or asthma were admitted to the CVW for disease optimisation and exacerbation management. Individualised management plans were delivered in the patients' home by hospital-based respiratory and community nursing teams, incorporating remote technology to monitor vital signs. Symptoms and health status at admission and discharge were compared.

Results Twenty patients were admitted. One-quarter of patients had asthma, 50\% COPD, and 25\% combined asthma/COPD. Patients had severe disease, mean (SD) $\mathrm{FEV}_{1}$ 50(20)\% predicted, and an average 6.4(5.7) exacerbations of disease in the previous 12 months. Patients received personalised disease and self-management education. All acute exacerbations $(n=11)$ were successfully treated in the community. The average length of CVW admission was 10(4) days. By discharge, $60 \%$ of COPD and $66 \%$ of asthma patients recorded improvements in symptoms score exceeding the minimal clinically important difference. Fifty percent had clinically meaningful improvements in health status.

Conclusion A CVW model facilitates the delivery of combined specialist and generalist care to patients with chronic respiratory disease in the community and improves symptoms and health status. The principles of the model are transferable to other conditions to improve overall health and reduce emergency hospital care.
\end{abstract}

Keywords Asthma $\cdot$ Chronic disease management $\cdot$ COPD $\cdot$ Integrated Care $\cdot$ Virtual Ward

Breda Cushen

bredacushen@beaumont.ie

1 Department of Respiratory Medicine, Beaumont Hospital, Dublin 9, Dublin, Ireland

2 Community Intervention Team, Community Healthcare Organisation Dublin North Central and County, Area 9 (CHO 9 DNCC), Dublin North City, Ireland

3 Office of the Nursing and Midwifery Services Director, Health Service Executive, Dublin South City, Ireland

4 Department of Health, Chief Nurse's Office, Dublin, Ireland

\section{Introduction}

Chronic respiratory diseases such as asthma and chronic obstructive pulmonary disease (COPD) are responsible for significant patient morbidity and mortality [1]. The true prevalence of these conditions in Ireland is unknown but it is estimated that almost $10 \%$ of adults have asthma [2] whilst more than 500,000 carry a diagnosis of COPD [2]. Ireland has one the highest rates of asthma and COPD hospitalisations in the world [3]. In 2017, 329 per 100,000 Irish adults were hospitalised for treatment of these conditions, far exceeding the OECD average of 225 hospitalisations per 100,000 adults [3]. For the most part, these hospitalisations are deemed to be "avoidable" and reflect inadequacies in 
the provision and access to specialist care in the community [3]. A revision of traditional models of care delivery in Ireland is needed in order to reduce the burden of these diseases on our healthcare system and to improve quality of life and outcomes for patients. The development of a more integrated and co-ordinated model of care across primary, community and social care, and acute hospitals is central to the Irish national long-term health and social care strategy, "Sláintecare"[4]. The added barriers to accessing care caused by the current SARS-CoV-2 pandemic have created an even greater urgency to instigate change.

The implementation of community virtual ward (CVW) initiatives to deliver combined specialist and generalist care to patients with chronic disease in their homes has increased over the last decade [5-7]. Several studies have demonstrated the effectiveness of CVWs in reducing unplanned hospital admissions and improving quality of life indices in frail elderly populations $[8,9]$. The benefit of CVW initiatives in identifying gaps in care with the potential to result in adverse events in patients undergoing home haemodialysis has also been described [10]. More recently, the CVW approach has been adopted to support patients with mild COVID-19 infection at home [11, 12]. The use of remote monitoring technology to record oxygen saturations has alleviated the pressure on overstretched acute hospital services by facilitating the discharge of patients whilst ensuring the onset of respiratory failure is detected and treated early in these community-based patients [13].

We undertook a proof-of-concept initiative to establish whether the integration of a CVW model of care into usual clinical practice was feasible and acceptable to patients and improved the clinical outcomes of patients attending our services with chronic respiratory disease.

\section{Methods}

This CVW initiative was implemented over 12 weeks between September and November 2020. This was an integrated model of care delivered by Beaumont Hospital and the HSE Community Healthcare Organisation Dublin North City and County Area 9 (CHO 9 DNCC). Two nursing teams were involved in the delivery of care to patients admitted to the CVW: a specialist respiratory team based at Beaumont Hospital, Dublin, Ireland, and a Community Intervention Nursing team (CIT) serving CHO 9 DNCC. CIT is a specialist and generalist nurse-led team that provides enhanced care for a defined period to patients based in the community with an acute episode of illness. The team includes senior clinical nursing leadership, enhanced nurses, and community registered general nurses serving the Beaumont Hospital catchment area. Clinical oversight and governance were provided by the respiratory consultant team at Beaumont Hospital with oversight for supportive care provided by CHO 9 DNCC.
Nominated GP practices within CHO9 DNCC and the Beaumont Hospital catchment area, and Beaumont Hospital respiratory services, were provided with information about the initiative and invited to refer patients directly to the CVW. All patients admitted to the CVW had a confirmed diagnosis of chronic airway disease (COPD and/or asthma) and were known to specialist respiratory services at Beaumont Hospital. The initial referral criteria included only those patients experiencing a mild-moderate exacerbation of their underlying respiratory disease who, following physical assessment by the referrer, were deemed suitable for community management. Due to limited numbers of face-to-face consultations and physical patient assessments taking place during the SARS-CoV-2 pandemic, initial referral numbers were low. As a result, inclusion criteria were broadened to include stable patients with poorly controlled disease i.e. $\geq 2$ community treated or $\geq 1$ hospital treated exacerbation in the previous 12 months, who had undergone either virtual or physical assessment by their GP or respiratory physician. All patients admitted to the CVW agreed to home visits and to the use of technology to remotely monitor their clinical status. An initial target recruitment of 20 admissions over 8 weeks was set. This recruitment period was extended by a further 4 weeks to ensure target recruitment was achieved.

Patients referred to the CVW underwent a virtual assessment of suitability by the hospital-based CVW team prior to admission. A comprehensive medical and respiratory history was obtained by the hospital team and following further assessment of the patient in their home by the CIT, and the completion of validated symptom and quality of life questionnaires, a personalised management plan was devised. Table 1 outlines the interventions offered to patients, the intensity of which varied depending on the individual patient need. The personalised disease and self-management

Table 1 Intervention types

Medication reconciliation

Social determinants of care

Anxiety management techniques

Inhaler technique

Education:

Disease education

Exacerbation management

Self-management planning

Smoking cessation

Airway clearance

Breathlessness management

Diet and nutrition

Bone health

Exercise

Vaccination 
education programme was delivered as a shared care approach integrating with community nursing (CIT). This was enabled by integration of referral processes, assessment of care needs, care plans, and regular ward rounds.

All patients were provided with remote monitoring technology which facilitated monitoring of daily oxygen saturations, heart rate, and spirometry measurements. Data was uploaded from a Bluetooth-enabled smartphone or tablet device to a password-protected patient and hospital portal, accessible only to registered CVW healthcare personnel. CIT staff provided a 7-day service to patients from 8 am to $8 \mathrm{pm}$. Out of hours support, where required, was provided to the CIT team by Beaumont Hospital nurse management, and on-call medical teams. Patients who became unwell after $8 \mathrm{pm}$ were advised to contact local emergency services. Patients were discharged from the CVW once exacerbation treatment, or disease optimisation interventions, were complete. Ongoing referral to appropriate outpatient specialist and community services was arranged on discharge on an individual, as-needed basis. Feedback on patient experience was obtained at time of discharge.

\section{Statistical analysis}

Data is presented as mean (standard deviation) or percent unless otherwise stated. Admission and discharge symptom and quality of life scores were compared using paired $t$-test. Analysis was performed using Stata v.13 (StataCorp, Texas, USA) with statistical significance set at $p<0.05$.

\section{Results}

\section{Baseline demographics}

Twenty patients were admitted to the CVW of whom $30 \%$ were referred by the GP. The remaining patients were referred directly from the respiratory outpatient clinic. One quarter of patients had a diagnosis of asthma, 25\% had combined asthma and COPD, and the remaining patients (50\%) had COPD. The majority of patients $(55 \%)$ were referred for disease optimisation, whilst $40 \%$ had a current exacerbation of airways disease. One patient with severe asthma was admitted to the CVW for facilitation of supervised oral corticosteroid wean. Co-morbidity was prevalent; there were a median 4.5 (5.5) additional diagnoses per person. The most common coexistent diagnoses were cardiovascular disease (70\%) and anxiety/depression (55\%). In addition to respiratory medications, an average 6.8 (5.3) medications were prescribed per person. Baseline demographic data is shown in Table 2.

\section{Disease severity}

Mean forced expiratory volume in one second $\left(\mathrm{FEV}_{1}\right)$ was $50(20) \%$ predicted. On average, patients had experienced 6.4 (5.7) exacerbations in the preceding 12 months with $65 \%$ requiring one or more hospital admissions for treatment, mean hospitalisation rate 1.6 (1.9) per person. All patients were prescribed maintenance inhaled therapy. Ninety percent of patients were prescribed a combined inhaled

Table 2 Baseline demographics $n=20$

\begin{tabular}{|c|c|c|c|}
\hline Age, years & $64.4(13.7)$ & Number of prescribed medications* & $6.8(5.3)$ \\
\hline Sex, male $(\%)$ & 35 & Maintenance ICS/LABA (\%) & 90 \\
\hline Smoking status (\%) & & Maintenance LABA/LAMA (\%) & 10 \\
\hline Current & 30 & Maintenance Azithromycin (\%) & 45 \\
\hline Ex-smoker & 55 & Leukotriene receptor antagonist $(\%)$ & 30 \\
\hline Pack year history & $46.6(26.5)$ & Maintenance prednisolone $(\%)$ & 5 \\
\hline Diagnosis $(\%)$ & & Nebulised short-acting bronchodilators (\%) & 55 \\
\hline Asthma & 25 & Stable State Spirometry & \\
\hline COPD & 50 & $\mathrm{FEV}_{1}(\mathrm{~L})$ & $1.3(0.7)$ \\
\hline Asthma-COPD overlap & 25 & $\mathrm{FEV}_{1}(\%$ predicted $)$ & $50.4(20.4)$ \\
\hline Number of co-morbidities & $5.3(3.7)$ & $\mathrm{FVC}(\mathrm{L})$ & $2.3(0.9)$ \\
\hline Cardiovascular disease $(\%)$ & 70 & $\mathrm{FEV}_{1} / \mathrm{FVC}$ & $51.7(14.5)$ \\
\hline Anxiety/depression (\%) & 55 & Long-term oxygen therapy requirement $(\%)$ & 25 \\
\hline Low bone mineral density (\%) & 25 & Number of exacerbations in last 12 months & $6.4(5.7)$ \\
\hline Diabetes mellitus (\%) & 15 & Community-treated & $4.0(3.6)$ \\
\hline Arrhythmia (\%) & 20 & Hospital admission & $1.6(1.9)$ \\
\hline \multirow[t]{3}{*}{ Gastro-oesophageal reflux (\%) } & 35 & Emergency department presentation & $1.1(1.9)$ \\
\hline & & Barthel Index, median (range) & $95(30-100)$ \\
\hline & & Rockwood Clinical Frailty Scale, median (range) & $4(2-6)$ \\
\hline
\end{tabular}

Data is presented as mean (standard deviation) unless otherwise stated

ICS inhaled corticosteroid, $L A B A$ long-acting beta-2-agonist, $L A M A$ long-acting muscarinic antagonist, $F E V_{l}$ forced expiratory volume in one second, $F V C$ forced vital capacity

*Non-respiratory medications 
corticosteroid/long-acting beta-2-agonist (ICS/LABA), of whom $75 \%$ were also prescribed long-acting muscarinic antagonist (LAMA) treatment. Just over half the cohort $(n=11)$ was also using regular nebulised short-acting bronchodilators. There was a high symptom burden at time of admission. COPD patients reported a mean COPD assessment test (CAT) [14] score of 24.3 (7.8) and modified Borg breathlessness score [15] of 1.9 (2.8). Mean modified Medical Research Council (mMRC) dyspnoea score was 2.6 (1.2), corresponding to breathlessness at $100 \mathrm{~m}$ or a few minutes mobilising on level ground [16]. The mean asthma control test (ACT) [17] score was 16 (5.9) consistent with suboptimal asthma control. Mean EQ-VAS [18] score was 53.5 (19.4) on a 100-point scale where 100 equates to best imaginable health status. Median Barthel Index [19] score was 95 (range 30-100) where 100 equates to full independence in activities of daily living. On average, patients were living with very mild frailty as determined by the Rockwood Frailty Scale [20], median score 4 (range 2-6).

\section{Interventions completed}

Inhaler technique was identified as suboptimal in $70 \%$ of patients and was corrected. Seven patients required a change in inhaler dosing or device change. Seven of the 11 patients taking home nebulised treatment reported side-effects of excess anti-muscarinic antagonist and beta-2-agonist treatment, and nebulised therapy was successfully discontinued. Basic education in airway clearance was provided to all patients. Seven patients (35\%) required referral to specialist physiotherapy services for ongoing education in airway clearance. Anxiety was identified as a significant contributor to symptom burden in $35 \%$ of patients all of whom were referred for psychology assessment. The majority of patients $(80 \%)$ agreed to onward referral to pulmonary rehabilitation.

\section{Clinical outcomes}

The average length of admission to the CVW was 10 days, range 5-24 days. All patients admitted to the CVW were successfully managed at home. Two patients had a new exacerbation during admission, both of whom continued treatment at home under the CVW team. One patient previously requiring hospital admission for a supervised wean of maintenance oral corticosteroid was successfully weaned following admission to the CVW from $10 \mathrm{mg}$ daily to $6 \mathrm{mg}$ daily without complication. Four patients required a physical assessment in the outpatient ambulatory unit by the hospitalbased team during their CVW admission. Hospital admission was avoided in each case. The out-of-hours escalation plan was not activated by any patient.

Symptom and quality of life scores were recorded at admission and discharge. Mean CAT score fell by 2.5 (7.2), $p=0.20$, at discharge from admission values, Fig. 1. Sixty percent of patients reported improvements in CAT exceeding the minimal clinically important difference of 2 points [21]. No substantial change in Borg breathlessness score or mMRC dyspnoea score was recorded. ACT improved by a mean of 3.17 (3.25), $p=0.06$, Fig. 1. In two-thirds of patients, improvements were greater than the MCID of the ACT of 3 points [22]. Overall health status, as determined by the EQ-VAS improved whilst on the CVW, with mean discharge EQ-VAS score of 65.4 (19.3), change of 11.85 (19.3), $p=0.055$, Fig. 1 . Of half the study population who recorded an improvement in health status, $90 \%$ exceeded the MCID for improvement in EQ-VAS score of 8 points [23].

\section{Patient experience}

Patient experience was established on discharge from the CVW using a modified version of the National Patient Experience Survey. Overall, feedback was positive from all patients. Common themes which emerged included:

i) Symptom recognition: greater understanding of selfmanagement including strategies to self-manage effectively and knowledge of supports available

ii) Remote monitoring: improved understanding of symptoms in the context of remote monitoring device results and reduced anxiety levels due to access to devices during the admission

iii) Shared care/partnership approach: appreciation for the integration of hospital and community teams and patient involvement in decision making

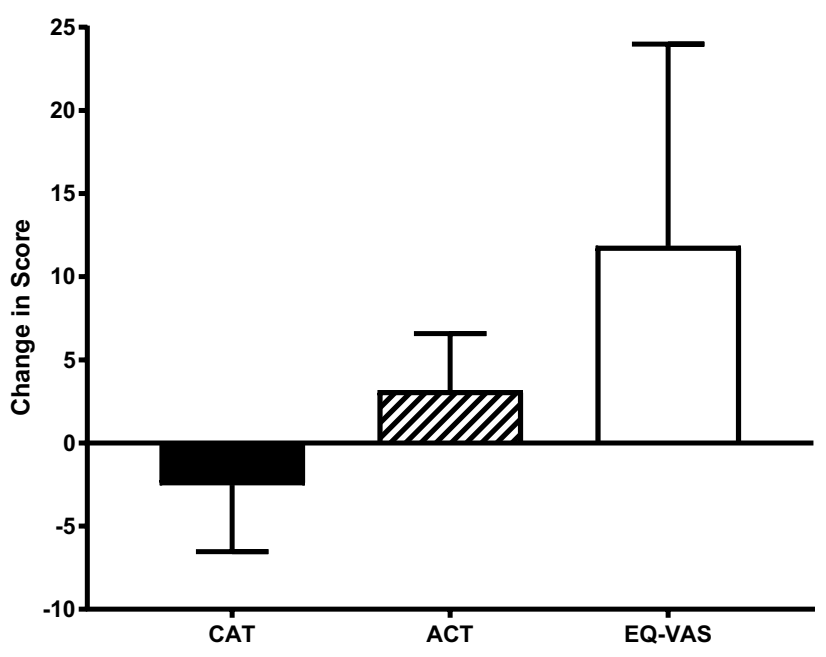

Fig. 1 Mean (95\% CI) change in COPD Assessment Test (CAT), Asthma Control Test (ACT), and EQ-VAS at time of discharge from the CVW. A mean change exceeding the minimal clinically important difference for each questionnaire was achieved 
iv) Care setting: expressed a preference for care at home and reported being less likely to present to the Emergency Department in the future and would leave hospital sooner, if there was access to a CVW

\section{Resource allocation and cost}

The CVW was delivered by staff within the current working structure of Beaumont Hospital and CIT based in CHO9 DNCC. One advanced nurse practitioner (ANP) and one candidate ANP based in the hospital setting shared care of patients admitted to the CVW between 8 am and 4 pm Monday to Friday and met weekly with the lead consultant to discuss cases. The CIT team included 2-3 enhanced nurses and one grade 1 clinical nurse manager (CNM1) per shift providing care between 8 am and 8 pm 7 days per week.

The number and duration of visits varied depending on individual patient needs and complexity. Additionally, as staff became more familiar with the process, the time spent completing assessments and the number of direct patient interactions reduced. The majority of care provided by the hospital-based team was remote care (i.e. delivered via phone or video consultation). Patients required on average 5.5 remote contacts lasting 47 min per consultation. All direct care provided by the CIT team was in the form of face-to-face home visits. The majority of patients were reviewed on admission, midway during admission, and on discharge. An average 3.5 visits were completed per patient with each visit taking, on average, $40 \mathrm{~min}$. The cost of admission to the CVW including the remote monitoring platform was estimated at 88 euro per patient per day versus an estimated 820 euro per day for an acute hospital admission.

\section{Discussion}

The Community Virtual Ward (CVW) initiative facilitated the delivery of integrated specialist and generalist multidisciplinary respiratory care to patients with severe chronic respiratory illness in their own homes. The core interventions delivered by the CVW team provided a blend of specialist and generalist care with targeted interventions to improve personalised disease education, self-management, social determinants of care, medication reconciliation, and adherence. Despite small numbers, the CVW interventions resulted in clinically significant improvements in symptoms and quality of life scores for the majority of patients and an enhanced patient experience of care delivery.

The patients recruited to the CVW had complex respiratory needs and a history of frequent healthcare use; twothirds had been admitted to hospital in the 12 months prior to admission. Given the increased morbidity and mortality associated with exacerbation events [24], these patients were deemed to be a vulnerable group who would benefit most from the initiative. In addition, all patients were known to hospital respiratory services; this ensured that the diagnosis of COPD and/or asthma was correct and had been confirmed by objective testing. In addition to addressing the core principles of good chronic disease management [25], the CVW team undertook a comprehensive assessment of each patient to identify factors contributing to suboptimal symptom control and burden of living with a chronic illness that increased frequent emergency healthcare use. Multi-morbidity is common in patients with chronic respiratory disease. The impact of factors extraneous to the primary respiratory diagnosis is often underestimated and can be difficult to appreciate during a brief consultation, or during a hospital presentation when management of the acute problem rightly takes precedence. However, it is well-recognised that comorbid factors contribute, often in a significant way, to disease burden and in many cases are the predominant driver of symptoms and emergency healthcare presentations [26-29]. A previous study of COPD patients admitted to Beaumont hospital identified a relationship between high anxiety scores, erratic adherence to maintenance inhaled therapy, and increased emergency department presentations [24]. Regular visits to the patient's home by the community intervention nursing team, combined with regular virtual interactions with specialist hospital staff, allowed the CVW team to gain a better understanding of the psychological, environmental, and social factors impeding a patient's ability to effectively manage their disease and, in turn, maintain good health. For example, high levels of co-existent depression and anxiety were identified which in several patients had a considerable influence on symptom burden and required ongoing specialist input.

Home visits were invaluable to identify barriers to medication adherence. Suboptimal adherence to maintenance inhaled therapy is well-recognised in chronic respiratory disease, as is the need for regular inhaler adherence assessment and education [30-32]. Attendance in the patients' home allowed for accurate medication reconciliation to take place which is not always possible in the outpatient clinic or GP visit setting. Significant polypharmacy was identified with an average seven prescribed medications per patient in addition to their respiratory medications and inhalers. All patients benefitted from the detailed medication review provided by the CVW staff. In $80 \%$ of cases, the team were able to rationalise nebulised therapy prescriptions and, following review of inhaler adherence and technique, changes were instituted in $40 \%$ and $70 \%$ of patients, respectively.

The provision of specialist support to the community teams by the hospital hub, aided by digital technology, empowered the non-specialist community teams to deliver personalised care to complex respiratory patients. This collaboration and integration of hospital specialist and community intervention teams created a shared learning experience for staff which in turn benefitted patients. The 
upskilling of all staff and the working relationships which are now established will continue to benefit patients into the future. The addition of remote monitoring technology ensured that any deterioration in clinical condition would be detected and acted upon in a timely manner. This combined approach using telemonitoring equipment to facilitate remote monitoring by the specialist hospital team and face-to-face interventions delivered by local community nursing teams allows patients with limited access to their specialist hospital team (e.g. as a result of physical or geographical barriers) to safely avail of this service. Challenges to using telemonitoring equipment were encountered as several patients did not have Wi-Fi capability; however, this did not hamper the ability of the CVW team to deliver high-quality care to these patients in their homes.

The generic framework of the CVW model could be easily replicated across other chronic diseases and for a variety of indications including management of acute pathologies and disease optimisation as has been demonstrated here. Additionally, the remit could be expanded to facilitate early supported discharge of patients from the acute hospital setting. The future sustainability of integrated programmes such as the CVW will however depend on the sufficient availability of both hospital and community staff to work full-time in the provision of chronic disease care without the competing interest of other acute service delivery. The CVW model of care compliments the integrated care programme for chronic disease in Ireland [33] which aims to improve access to specialist care for patients with chronic disease in the community through the establishment of specialist community hubs.

Standardising processes of care and documentation were essential to integration, and the availability of a portal to share information across the hospital and community ensured all patient data, and assessments were accessible to all staff in real-time. This allowed for seamless handover of patients and facilitated staff leave and/or less than full-time staff to work on the CVW team without incident or interruption in patient care. The provision of robust IT infrastructure and good information governance to support shared and integrated care between hospital and community teams is integral to the successful implementation of similar initiatives in the future. Given the short timeframe, we were unable to assess the impact of the CVW on emergency hospital presentation rates, but this will be examined in the future.

\section{Conclusion}

A community virtual ward model of care is feasible and acceptable to patients with chronic respiratory disease and has a positive impact on both symptoms and patient-reported health status. The additional knowledge gained from assessing patients within their home environment ensures the development, and implementation, of personalised management plans maintaining patients at the centre of care.

Acknowledgements We would like to thank the patients admitted to the CVW who provided feedback on this new service. We wish to acknowledge and thank the Chief Nurse's Office in the Department of Health, Office of the Nursing and Midwifery Services Director in the Health Service Executive, the staff of Beaumont Hospital Respiratory Department, Meabh Smith, Clinical Engineering department, Beaumont Hospital, and the Beaumont Hospital out-of-hours nursing team and staff of the Community Healthcare Organisation Area (9) Dublin North Central and County.

Author contribution CL, RC, AM, DC, and MEOB designed the CVW structure and protocols. All authors were involved in service implementation. Material preparation, data review, and analysis were performed by BC. The first draft of the manuscript was written by BC and CL. All authors critically revised and approved the final version of the manuscript.

Availability of data and material Available on request.

\section{References}

1. Hay S (2017) Global, regional, and national deaths, prevalence, disability-adjusted life years, and years lived with disability for chronic obstructive pulmonary disease and asthma, 1990-2015: a systematic analysis for the Global Burden of Disease Study 2015. Lancet Respir Med 5:(9)

2. Irish Thoracic Society (2018) Respiratory health of the nation.

3. OECD (2019) (2019) Health at a glance 2019: OECD Indicators, OECD Publishing.

4. SlainteCare. https://www.gov.ie/en/campaigns/slaintecareimplementation-strategy/. Accessed 26/04/2021 2021

5. Jones J, Carroll A (2014) Hospital admission avoidance through the introduction of a virtual ward. Br J Community Nurs 19(7):330-334

6. Uminski K, Komenda P, Whitlock R, Ferguson T, Nadurak S, Hochheim L, Tangri N, Rigatto C (2018) Effect of post-discharge virtual wards on improving outcomes in heart failure and nonheart failure populations: a systematic review and meta-analysis. PLoS ONE 13(4):e0196114

7. Lewis G, Vaithianathan R, Wright L, Brice MR, Lovell P, Rankin S, Bardsley M (2013) Integrating care for high-risk patients in England using the virtual ward model: lessons in the process of care integration from three case sites. Innt J Integr Care 13

8. Lewis C, Moore Z, Doyle F, Martin A, Patton D, Nugent LE (2017) A community virtual ward model to support older persons with complex health care and social care needs. Clin Interv Aging 12:985

9. Leung DY, Lee DT-F, Lee IF, Lam L-W, Lee SW, Chan MW, Lam Y-M, Leung S-H, Chiu P-C, Ho NK (2015) The effect of a virtual ward program on emergency services utilization and quality of life in frail elderly patients after discharge: a pilot study. Clin Interv Aging 10:413

10. Nadeau-Fredette A-C, Chan CT, Bargman JM, Copland MA, Finkle SN, Oliver MJ, Pauly RP, Perl J, Shah NA, Zimmerman DL (2019) Predictors of care gaps in home dialysis: the home dialysis virtual ward study. Am J Nephrol 50(5):392-400 
11. Ferry OR, Moloney EC, Spratt OT, Whiting GF, Bennett CJ (2021) A virtual ward model of care for patients with COVID19: retrospective single-center clinical study. J Med Internet Res 23(2):e25518

12. Thornton J (2020) The "virtual wards" supporting patients with COVID-19 in the community. BMJ (Online) 369

13. O'Carroll O, MacCann R, O'Reilly A, Dunican EM, Feeney ER, Ryan S, Cotter A, Mallon PW, Keane MP, Butler MW (2020) Remote monitoring of oxygen saturation in individuals with COVID-19 pneumonia. Eur Respir J 56:(2)

14. Jones P, Harding G, Berry P, Wiklund I, Chen W, Leidy NK (2009) Development and first validation of the COPD Assessment Test. Eur Respir J 34(3):648-654

15. Borg GA (1982) Psychophysical bases of perceived exertion. Med Sci Sports Exerc 14(5):377-381

16. Stenton C (2008) The MRC breathlessness scale. Occupational medicine (Oxford, England) 58(3):226-227. https://doi.org/10. 1093/occmed/kqm162

17. Nathan RA, Sorkness CA, Kosinski M, Schatz M, Li JT, Marcus P, Murray JJ, Pendergraft TB (2004) Development of the asthma control test: a survey for assessing asthma control. J Allergy Clin Immunol 113(1):59-65

18. Rabin R, Charro Fd (2001) EQ-SD: a measure of health status from the EuroQol Group. Ann Med 33(5):337-343

19. Mahoney FI, Barthel DW (1965) Functional evaluation: the Barthel Index: a simple index of independence useful in scoring improvement in the rehabilitation of the chronically ill. Maryland state medical journal

20. Rockwood K, Song X, MacKnight C, Bergman H, Hogan DB, McDowell I, Mitnitski A (2005) A global clinical measure of fitness and frailty in elderly people. CMAJ 173(5):489-495

21. Kon SS, Canavan JL, Jones SE, Nolan CM, Clark AL, Dickson MJ, Haselden BM, Polkey MI, Man WD (2014) Minimum clinically important difference for the COPD Assessment Test: a prospective analysis. Lancet Respir Med 2(3):195-203. https://doi. org/10.1016/s2213-2600(14)70001-3

22. Schatz M, Kosinski M, Yarlas AS, Hanlon J, Watson ME, Jhingran $\mathrm{P}$ (2009) The minimally important difference of the Asthma Control Test. J Allergy Clin Immunol Pract 124 (4):719-723. e711

23. Zanini A, Aiello M, Adamo D, Casale S, Cherubino F, Della Patrona S, Raimondi E, Zampogna E, Chetta A, Spanevello A (2015) Estimation of minimal clinically important difference in EQ-5D visual analog scale score after pulmonary rehabilitation in subjects with COPD. Respir Care 60(1):88-95

24. Cushen B, Sulaiman I, Greene G, MacHale E, Mokoka M, Reilly RB, Bennett K, Doyle F, van Boven JFM, Costello RW (2018) The clinical impact of different adherence behaviors in patients with severe chronic obstructive pulmonary disease. Am J Respir Crit Care Med 197(12):1630-1633. https://doi.org/10.1164/rccm. 201712-2469LE
25. Wagner EH, Austin BT, Von Korff M (1996) Organizing care for patients with chronic illness. The Milbank Quarterly:511-544

26. Hew M, Menzies-Gow A, Hull JH, Fleming L, Porsbjerg C, Brinke AT, Allen D, Gore R, Tay TR (2020) Systematic assessment of difficult-to-treat asthma: principles and perspectives. J Allergy Clin Immunol Pract. https://doi.org/10.1016/j.jaip.2020. 02.036

27. Porsbjerg C, Menzies-Gow A (2017) Co-morbidities in severe asthma: clinical impact and management. Respirology (Carlton, Vic) 22(4):651-661. https://doi.org/10.1111/resp.13026

28. Westerik JA, Metting EI, van Boven JF, Tiersma W, Kocks JW, Schermer TR (2017) Associations between chronic comorbidity and exacerbation risk in primary care patients with COPD. Respir Res 18(1):31. https://doi.org/10.1186/s12931-017-0512-2

29. Gudmundsson G, Gislason T, Janson C, Lindberg E, Hallin R, Ulrik CS, Brondum E, Nieminen MM, Aine T, Bakke P (2005) Risk factors for rehospitalisation in COPD: role of health status, anxiety and depression. Eur Respir J 26(3):414-419. https://doi. org/10.1183/09031936.05.00078504

30. Sulaiman I, Cushen B, Greene G, Seheult J, Seow D, Rawat F, MacHale E, Mokoka M, Moran CN, Sartini Bhreathnach A, MacHale P, Tappuni S, Deering B, Jackson M, McCarthy H, Mellon L, Doyle F, Boland F, Reilly RB, Costello RW (2017) Objective assessment of adherence to inhalers by patients with chronic obstructive pulmonary disease. Am J Respir Crit Care Med 195(10):1333-1343. https://doi.org/10.1164/rccm. 201604-0733OC

31. O'Dwyer S, Greene G, MacHale E, Cushen B, Sulaiman I, Boland F, Bosnic-Anticevich S, Mokoka MC, Reilly RB, Taylor T, Ryder SA, Costello RW (2020) Personalized biofeedback on inhaler adherence and technique by community pharmacists: a cluster randomized clinical trial. J Allergy Clin Immunol Pract 8(2):635-644. https://doi.org/10.1016/j.jaip.2019.09.008

32. Sulaiman I, Greene G, MacHale E, Seheult J, Mokoka M, D’Arcy S, Taylor T, Murphy DM, Hunt E, Lane SJ, Diette GB, FitzGerald JM, Boland F, Sartini Bhreathnach A, Cushen B, Reilly RB, Doyle F, Costello RW (2018) A randomised clinical trial of feedback on inhaler adherence and technique in patients with severe uncontrolled asthma. The Eur respir J 51 (1). https://doi.org/10.1183/ 13993003.01126-2017

33. ICP for Prevention and Management of Chronic Disease. https:// www.hse.ie/eng/about/who/cspd/icp/chronic-disease/. Accessed 26/04/2021 2021

Publisher's Note Springer Nature remains neutral with regard to jurisdictional claims in published maps and institutional affiliations. 